\title{
Assessing the Eligibility of Kidney Transplant Donors
}

\author{
Francisco Reinaldo ${ }^{1}$, Carlos Fernandes ${ }^{1}$, Md. Anishur Rahman ${ }^{4}$, Andreia \\ Malucelli $^{3}$, and Rui Camacho ${ }^{4}$ \\ 1 FEUP, Universidade do Porto \\ Rua Dr. Roberto Frias, sn, 4200-465 Porto, POrtugal \\ and \\ UnilesteMG - Centro Universitário do Leste de Minas Gerais \\ GIC - Grupo de Inteligência Computacional \\ Av. Presidente Tancredo Neves n. 3500, Bairro Universitário, CEP 35170-056 \\ Coronel Fabriciano, MG, Brasil \\ reifeup@fe.up.pt \\ 2 Pontifical Catholic University of Paraná - PUCPR, \\ PostGraduate Programme in Health Technology - PPGTS \\ R. Imaculada Conceição, 1155 \\ 215-901 Curitiba PR, Brazil \\ carlos.fernandes@pucpr.br \\ 3 Pontifical Catholic University of Paraná - PUCPR, \\ PostGraduate Programme in Health Technology - PPGTS \\ R. Imaculada Conceição, 1155 \\ 80215-901 Curitiba PR, Brazil \\ malu@ppgia.pucpr.br \\ 4 FEUP, Universidade do Porto, \\ Rua Dr Roberto Frias s/n, 4200-465 Porto, Portugal
}

\begin{abstract}
Organ transplantation is a highly complex decision process that requires expert decisions. The major problem in a transplantation procedure is the possibility of the receiver's immune system attack and destroy the transplanted tissue. It is therefore of capital importance to find a donor with the highest possible compatibility with the receiver, and thus reduce rejection. Finding a good donor is not a straightforward task because a complex network of relations exists between the immunological and the clinical variables that influence the receiver's acceptance of the transplanted organ. Currently the process of analyzing these variables involves a careful study by the clinical transplant team. The number and complexity of the relations between variables make the manual process very slow. In this paper we propose and compare two Machine Learning algorithms that might help the transplant team in improving and speeding up their decisions. We achieve that objective by analyzing past real cases and constructing models as set of rules. Such models are accurate and understandable by experts.
\end{abstract}




\section{Introduction}

Organ transplantation can bring many benefits for individuals suffering from a variety of incurable diseases. Transplantation is an alternative medical solution to restore damaged functions in patients, thereby offering improved quality of life [1].

In recent years surgeries in which an organ or tissue is transplanted have seen a major increase in Brazil. In 2005, 15,527 organ and tissue transplants were successfully performed. This represents an 18,3\% increase over 2003 figures. This growth is a consequence of an increased awareness among the Brazilian population, the competent performance of teams and institutions authorized by the Ministry of Health and the regulation of the National Transplant System (NTS) in accordance with prevailing legislation [2]. However, whilst the number of transplants has increased, the number of clinical transplant teams remains the same and the excessive workload may reach critical levels, making it more difficult to select the best donor for a given receiver. Organ transplantation requires a high degree of certainty and special care when making decisions, since the receivers immune system may attack and destroy transplanted tissue (organ rejection).

It is therefore necessary to find a donor who has the highest possible compatibility with the receiver, and thus reduce rejection [3]. This is not a straightforward task because a variety of immunological and clinical variables influence the receiver's acceptance of the transplanted organ. These variables may not be examined on a one by one basis but rather in an holistic manner, whereby one variable can interfere with another, potentiating or not the influence of each variable on the immune system. There is a complex relation between these variables. This network of relations is not entirely understood by the medical doctors.

Currently the process of analyzing these variables involves a careful study by the clinical transplant team. As this is a manual process, it is also a slow one owing to the number of variables and the need to establish the influence relationship between them. This comparison is detailed and slow because the specialist has to compare the receiver's entire history with possible donors, which requires specialized knowledge acquired through previous examples and continuous studies in the domain area. The clinical team uses previous cases to improve their knowledge and understanding concerning the decision on if a transplant should be performed or not. In our study we consider the use of Machine Learning (ML) algorithms to help the clinical transplant team. In the line of the human experts procedure the ML algorithms use a set of past cases (training examples) to construct models capable of correctly predict the outcome of unseen patient cases.

In this paper we describe the use of a rule induction algorithm $(\mathrm{CN} 2[4,5])$ and an Inductive Logic Programming (ILP) system[6] to construct the models that should help the transplant team decision process. We have used a database containing real information on previous transplants (receivers, donor and organ survival following transplant). The database used in the experiments is from the 
Pontifical Catholic University of Paraná, located in the city of Curitiba, in the state of Paraná in southern Brazil. The immunogenetics laboratory referred to is a national reference due to the volume of tests carried out and the quality of the techniques used. It is also a reference for the training of specialists in the field of immunogenetics. The models constructed by the ML algorithms achieved $77 \%$ accuracy.

This paper is structured as follows. Section2 presents a brief overview of kidney transplantation in Brazil, with emphasis on clinical and immunological evaluations. Rule induction and ILP techniques for extracting rules for correct decision making are explained in Section 3. Section 4 describes the experiments done and the results obtained. Section 5 concludes the paper and presents perspectives for future work.

\section{Kidney Transplantation}

Organ transplantation is a treatment procedure of great medical importance for thousands of people. Kidney transplantation is recommended in cases of chronic kidney failure at the terminal stage [7], when the receiver is undergoing dialysis or even at the pre-dialysis stage (pre-emptive transplantation) [8].

Transplantation can be offered to all kidney transplant candidates as an alternative aimed at reducing the incidence of vascular, cardiac, ocular and neurological complications inherent in diabetes [9]. Kidney transplantation is an important treatment option for the receiver both from the medical, and also from the social and economic point of view [10]. A Federal Decree regulates kidney transplants from live donors in Brazil [11]. The decree regulates Law No. 9,434, which makes provision for the removal of organs, tissues and parts of the human body for the purpose of transplantation and treatment. Pre-operation evaluations are necessary in order to verify the possibility of transplantation being performed and must take into consideration, for example, the duration of dialysis; the receiver's history; blood characteristics (haemoglobin, coagulation, biochemistry, ABO and immunology) as well as a variety of other examinations (see [12] for a detailed description).

\subsection{Clinical Evaluation}

Nowadays there are few contraindications for kidney transplantation. The receiver's age is no longer a reason for contraindication, as it was until relatively recently. Currently transplants are performed on newborn babies, including premature babies, and also on receivers more than 70 years old [10].

\subsection{Immunological Evaluation}

Once the receiver has had all the clinical examinations, he or she then undergoes a series of immunological examinations. The purpose of these examinations is to assess the survival of the organ and the receiver for as long as possible, 
thus promoting the length and the quality of the receiver's life, as well as avoiding wasting such a precious and scarce resource as an organ available for transplantation. In order to determine the histocompatibility between the receiver and the donor a study is made of the HLA (Human Leukocyte Antigens) complex, the principal characteristic of which is the high degree of polymorphism, which is found in a variety of cells [3]. Determining a receiver's HLA is important for verifying the prospects of clinical recovery or whether the receiver will present chronic HLA associated diseases [13]. Currently a variety of sites perform analysis on HLA molecules owing to their conformation, using programs such as HLA Matchmaker which indicates acceptable mismatches between receiver and donor, principally in the case of receivers who are hypersensitive to transplantation [14]. Pre-operation immunological evaluation must also take into consideration antibody panel tests to assess the strength of the reaction and the characterization of the antibodies in circulation [15].

\section{A Machine Learning Approach}

In order to help the transplant team to better understand what makes a transplant successful, we have analyzed a data set of real cases using two Machine Learning (ML) algorithms. We have applied a propositional learner, CN2 to induce production rules, and an Inductive Logic Programming (ILP) system. The justification for our choice is based on the fact that we need a symbolic model to present to the expert (this excludes Artificial Neural Networks and statistical approaches). It is well known in Artificial Intelligence (AI) and psychology that production rules are a formalism that humans comprehend easily. We have also included ILP in the set because ILP is known to perform well with small data sets and to produce very simple models. We therefore compare the two alternatives in the construction of simple models to help the transplant team to understand better what makes kidney transplants successful. Apart from producing a comprehensible model ML algorithms may be used to indicate which are the most relevant variables to determine the outcome of transplantation procedures.

A main objective of $\mathrm{CN} 2[4]$ is to assist users in making correct decisions, enabling new or modified theories to be derived through observation. In this sense, CN2 tries to discover patterns in a data set and returns the "best" set of decision rules understandable by human beings.

The CN2 Induction algorithm was developed at the Turing Institute as part of the Machine Learning Toolbox project. The algorithm was designed to be an efficient induction tool of simple decision rules in domains where there might be noise (errors due to transcription or errors due to an insufficient description language) $[16,4]$. This algorithm was previously built for a Unix platform but new improvements have also made CN2 available for Microsoft Windows platforms [17]. Since we are dealing with real data that has, possibly, some misclassified records and missing values, we need a robust ML tool such as CN2. 
Basically the CN2 mechanism works as follows:

Observation of the facts Two data files are loaded to obtain and test the decision rules: a file with attribute and class information and; the examples file;

Discovery of a relationship between them In an iterative way, the algorithm searches for the conditions that enable the "explanation" of a large number of samples belonging to the same class. The concept description language is of the form:

IF <complex> THEN predict <class>.

A <complex $>$ is specialized by either adding a new conjunctive term or removing a disjunctive element in one of its selectors. The learning algorithm works in an iterative way, where for each new iteration the algorithm searches for a <complex> that examines a large number of samples in a unique class <C> and few in other class. The system searches the <complex $>$ by performing a pruned global-to-local search;

Generalization The system performs a general-to-specific search with pruning. When a <complex $>$ is evaluated as good, the samples explained are removed from the training set and the rule IF <complex> THEN predict $\mathrm{C}$ is added to the end of the rule list. The last rule in the CN2 list is a "default rule" that classifies the all new samples based on the frequent class. This process is repeated until the satisfactory <complex> no longer exists.

The latest improvements to the rule syntax include the possibility of expressing a rule in the form: IF ...THEN ...ELSEIF ... THEN ..., called rule lists [18]. The important point is that the semantics of each individual rule depend on the previous ones, i.e., to trigger a rule, all the previous rules must have failed. This feature of the rule list excludes the possibility of a clash during the classification process but makes it more difficult to be interpreted by humans.

Inductive Logic Programming is a major field in Machine Learning with important applications in Data Mining. The fundamental goal of a predictive ILP system is to construct models (usually called hypotheses) given background knowledge $B$ and observations (usually called examples in the ILP literature) $E$. It is usual to have two kinds of examples: positive examples $\left(E^{+}\right)$(instances of the target concept) and negative examples $\left(E^{-}\right)$(used to avoid overgeneralization). A characteristic of ILP is that both data and models (hypotheses) are expressed in a subset of First Order Logic providing and expressive language to encode data and hypotheses.

The problem that a predictive ILP system must solve is to find a consistent and complete model $H$, in the form of a set of clauses, i.e., find a set of clauses that explain all given positive examples, while being consistent with the given negative examples. Since it is not usually obvious which set of clauses should be considered for inclusion in a theory, an ILP system must search through a very large space of hypotheses to find a set with the desired properties. See $[19,20]$ for a detailed survey on ILP.

In our experiments we used a well known ILP system called Aleph [6]. 


\section{Experiments}

\subsection{Experimental Settings}

The undertaken experiments address two questions: (Question 1) are the expert provided discretization values adequate for a propositional learner to achieve good performance? and ; (Question 2) can we provide an algorithm that does not require discretization of values and produces a simple and accurate model?

To answer these two questions we used a data set of 106 real cases of kidney transplant characterized by 12 attributes as shown in Table 1 . The original transplant problem is a regression one where the class value is the number of months the transplant patient survived. We have converted the problem into a two class problem by establishing a threshold on the numerical class values. Above 60 months we considered that the transplantation was successful and below that value it was considered not successful. The threshold value was provided by an expert. With the specified threshold there were 29 successful transplants and 79 not successful ones. We conducted two sets of experiments to answer the questions stated above.

In a first set we used the advise of an expert and discretized all numerical attributes. We used CN2 with a 5-fold cross validation to analyze that data set. We have tuned the CN2 parameters and the best results were obtained with a star o size 15 .

In the second set of experiments we used the Aleph ILP system to analyze the raw data . Again we used a 5-fold cross validation procedure. We have also tuned the Aleph system's minpos, noise and minaccuracy parameters. The final theory was constructed using the following settings: noise $=4$, minaccuracy $=$ 0.8 and $\operatorname{minpos}=4$.

Table 1. The attributes of the transplant data set file.

\begin{tabular}{ll}
\hline Attribute & type/possible values \\
\hline patient_gender & male, female; \\
$\begin{array}{l}\text { donor_gender } \\
\text { relationship }\end{array}$ & male, female; \\
patient_age & integer; \\
donor_age & integer; \\
compatibility_abo & yes, no, not_informed; \\
compatibility_hla_a & one_compatibility, none, two_compatibilities; \\
compatibility_hla_b & one_compatibility, none, two_compatibilities; \\
compatibility_hla_dr one_compatibility, none, two_compatibilities; & oner, father, unrelated; \\
crossmatch & positive, negative; \\
pregnancies_patient & integer; \\
pregnancies_donor & integer;
\end{tabular}




\subsection{Experimental Results}

The results are shown in Table 2. The first line concerns the use of CN2 with expert provided discretization values. The results achieved by $\mathrm{CN} 2$ are below a naive classifier that used the majority class. The result suggests that we should not rely on expert information concerning discretization of attribute values. We should wither use the raw data or use some ML method to descritize the values. The answer to Question 1 is therefore negative: we did not perform good using the exert suggested discretization values. Another negative result obtained with the first set of experiments is that the model is not simple (34 rules).

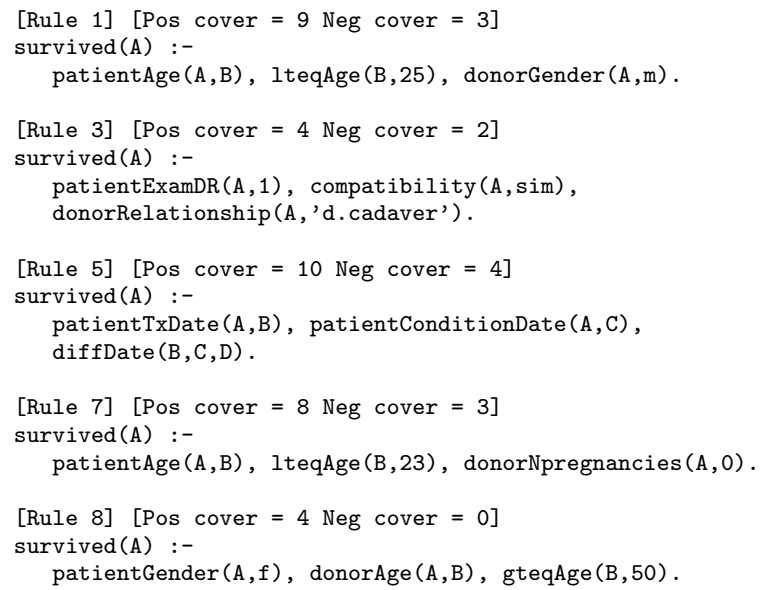

Fig. 1. Rules constructed by Aleph

On the other hand we can answer positively to Question 2: we can propose and algorithm that used the raw data and improves over the naive classifier that uses the majority class. Aleph not only achieve a better performance but also produced a very simple model with just 5 rules (see Figure 1). A careful analysis of the rules indicate that we have a lot to improve. Some of the rules (like rules 1 and 8) would not appear in a large size sample, they have a fair coverage possibly because of coincidences in the small number of positive cases. From the expert point of view the only interesting rule is rule 3 that uses blood type information and exams values.

\section{Conclusions and Future Work}

In the work reported in this paper we addressed a complex and very useful problem, that of helping a kidney transplant team to improve the success rate 
Table 2. Accuracy (Acc.) and number of rules (n. rules) of the constructed models. Majority class predictor is $74.5 \%$.

\begin{tabular}{cccc}
\hline Question & Algorithm & Acc. & n. rules \\
\hline Q1 & CN2 & $72.6 \%$ & 34 \\
Q2 & Aleph & $77 \%$ & 5
\end{tabular}

of organ transplant. We have applied ML learning algorithms to real cases of kidney transplant. We have achieved the construction of a very simple model with an significant accuracy improvement over a naive classifier.

However we still need to improve considerably usefulness of the model in order to be helpful to the transplant team. For that purpose we will collect more data. We have also to include further constraints in the ILP system in order to include a larger number of relations that the expert finds informative.

\section{Acknowledgements}

This work has been partially supported by the project ILP-Web-Service (PTDC/EIA/70841/2006) and by Fundação para a Ciência e Tecnologia. Francisco Reinaldo is funded by FCT grant SFRH/BD/17051/2004. Md. Anishur Rahman is funded by a FCT grant from project PTDC/BIA-PRO/72838/2006.

\section{References}

1. Altman, L.: Distúrbios imunes transplante (disturb immune transplant). In: Manual merck. (2006) http://www.msd.brazil.com.

2. SNT. In: Sistema nacional de transplantes (National Transplant System). (2007)

3. Abbas, A.: Imunologia Celular e Molecular (Cellular and Molecular Immunology). 3rd edn. Revinter (2000)

4. Clark, P., Boswell, R.: Rule induction with CN2: Some recent improvements. In: Proc. Fifth European Working Session on Learning, Springer-Verlag (1991) 151-163

5. Clark, P., Niblett, T.: The cn2 induction algorithm. Machine Learning 3(4) (1989) 261-283

6. Srinivasan, A.: Aleph manual

7. Fitzwater, D.: The outcome of renal transplantation in children without prolonged pre-tranplant dialysis. Clinical Pediatrics 30 (1991) 148-152

8. R.A.Wolfe: Comparison of mortality in all patients on dialysis awaiting transplantation, and recipients of a first cadaveric transplant. The New England Journal of Medicine 314 (1999) 1725-1730

9. Berthoux, F.: Pre-emptive renal transplantation in adults aged over 15 years. Nephrology Dialysis Transplantation 11 (1996) 41-43

10. Noronha, I.L.: Diretrizes em transplante renal (guidelines on renal transplantation). (2006) http://www.sbn.org.br/Diretrizes/tx.

11. DF: Decreto federal num. 2.268 (federal decree issue 2,268). (30th June 1997)

12. Baptista-Silva, J.: Transplante renal: cirurgia no receptor: adulto (2003) http: //www. lava.med.br/livro. 
13. Antunes, L.: Imunologia Geral (General Immunology). Atheneu (1999)

14. Duquesnoy, R.: Hlamatchmaker: a molecularly based algorithm for histocompatibility determination. Human Immunology 63 (2002) 339-352

15. Fernando, M.: Hlamatchmaker: a molecularly based algorithm for histocompatibility determination. Revista Brasileira de Cirurgia Cardiovascular (Brazilian Journal of Cardiovascular Surgery) 16 (2001)

16. Clark, P., Niblett, T.: Induction in noisy domains. In: Progress in Machine Learning-Proceedings of EWSL 87: 2nd European Working Session on Learning. (1987) 11-30

17. Reinaldo, F., Siqueira, M.: CN2 for microsoft windows XP (2006)

18. Rivest, R.L.: Learning decision lists (1987)

19. Muggleton, S.: Inductive logic programming. In: Proceedings of the 1st Conference on Algorithmic Learning Theory. 43-62

20. Muggleton, S., Raedt, L.D.: Inductive logic programming: Theory and methods. Journal of Logic Programming 19,20 (1994) 629-679 